\title{
Treatment of psychosis in Parkinson's disease
}

\author{
Denise Duncan and David Taylor
}

Psychosis is often associated with Parkinson's disease. The cause of the psychosis cannot usually be confirmed but it may be due to disease progression or, more commonly, to the drugs used in the management of Parkinson's disease.

The drug treatment of Parkinson's disease usually centres on improving dopaminergic function in the nigro-striatal pathways. Levo-dopa and selegiline increase the amount of synaptic dopamine available; apomorphine, bromocriptine and pergolide are agonists at dopamine receptors. Symptoms may also be improved by redressing the balance of dopaminergic and cholinergic activity. Benzhexol, procyclidine and orphenadrine, among others, are antagonists at muscarinic receptors. All of these drugs have been associated with psychiatric phenomena. This is not surprising since they can be said to mimic pathological processes: schizophrenia may be associated with increased dopamine activity; dementia with decreased acetylcholine activity.

If psychosis occurs and is severe or long-lived, then drug withdrawal may be contemplated. Generally, the last anti-Parkinsonian drug to be prescribed before the problem arose should be stopped first. Otherwise the following order for stopping drugs should usually be followed: anticholinergics, selegiline, amantadine, dopamine agonists (Quinn, 1995). After this, the dose of levo-dopa should be gradually reduced. Some success may be gained by manipulating the timing and size of levo-dopa doses. In particular, giving levo-dopa after food may slow its absorption and diminish adverse psychiatric effects.

Treating psychosis by these methods can be problematic, as withdrawing the offending agent may lead to loss of motor control. Treating movement disorders associated with Parkinson's disease while avoiding psychiatric ill-effects is therefore a delicate process.

Recent studies have suggested that apomorphine may be the anti-parkinsonian drug of choice in patients who develop psychosis while on oral anti-Parkinsonian treatment (Ray Chaudhuri et al, 1990; Ellis et al, 1995). In the open study by Ellis et al, twelve patients who developed problematic neuropsychiatric complications with their oral anti-Parkinsonian medication were treated with subcutaneous apomorphine for a mean period of $7.2 \pm 3$ months (range 2-14). Two of these patients discontinued therapy (one because of nausea despite domperidone treatment and one because of an increase in the number of falls). In the remaining patients apomorphine was continued (mean clinically effective dose: $58.8 \pm 41.3 \mathrm{mg} / 24$ hours (range 25-140) ), levo-dopa doses were reduced and oral dopamine agonists discontinued. A reduction or discontinuance of previous psychiatric problems was observed in all patients and no new psychiatric problems were noted. The authors postulated that this improvement in neuropsychiatric symptoms seen in these patients may be because apomorphine has an antipsychotic action.

If the above measures are not successful, a neuroleptic may be considered. As all standard neuroleptics are dopamine antagonists and can cause movement disorders, their use is far from ideal. Nevertheless, they are often required in the clinical situation. Those drugs which are less likely to cause extrapyramidal side-effects, such as thioridazine or sulpiride, should be tried first. Only low doses should be used (Quinn, 1995). Another option is risperidone, an antipsychotic with atypical features, which is known to cause fewer extrapyramidal side-effects than classical neuroleptics. In one open-label trial, six patients with Parkinson's disease and levo-dopa-induced hallucinations were treated with risperidone (Meco et al, 1994). Doses of up to $1.25 \mathrm{mg}$ per day were used and patients were treated for up to 24 weeks. The risperidone was well tolerated and hallucinations improved significantly in all patients. In contrast, a second study of six patients with Parkinson's disease found that all patients had worsening Parkinsonism (Ford et al, 1994). In this study the average daily dose of risperidone was $1.5 \mathrm{mg}$.

To date, clozapine seems to be the most extensively studied antipsychotic in Parkinson's disease, with numerous case reports and openlabel trials reported. In the majority of cases, patients experienced an improvement or even 
resolution of psychotic symptoms without a deterioration of motor function. Doses of between 6.25 and $100 \mathrm{mg} /$ day were used in open-label trials and the adverse effects reported were similar to those experienced in patients without Parkinson's disease. Limitations to these trials included rating scales not being consistently used (assessments were often subjective), a lack of information on concomitant medication, small sample numbers and short follow-up periods (Pfeiffer et al, 1994).

Two long-term studies of the use of clozapine in Parkinson's disease have now been reported. The first study (Factor et al, 1994) of 19 patients (two dropped out within two months) initially began patients on clozapine $25 \mathrm{mg}$ at bedtime. Because the first eight patients experienced excess sedation, the eleven patients that followed were started on $12.5 \mathrm{mg}$ a day. Subjects were treated for 6 to 24 months (mean 15 months) and they were evaluated using well-known psychiatric and Parkinson's disease rating scales.

While the subjects were taking clozapine, their mental state improved. Levo-dopa doses could therefore be increased and an improvement in Parkinsonian symptoms resulted. Two patients were also then able to tolerate the addition of levodopa when previous challenges had caused psychosis. Generally, clozapine doses of no greater than $50 \mathrm{mg}$ a day were prescribed as higher doses resulted in sedation and confusion. Other adverse effects experienced included drooling (ten patients), orthostatic hypotension, palpitations and a worsening of Parkinsonism (one patient each). During the second year of the study, the improvement in subjects' mental state did not remain statistically significant. This was possibly due to higher doses of levo-dopa being used, worsening dementia and the inability of subjects to tolerate higher clozapine doses.

In the second study (Rabey et al, 1995), 27 Parkinson's disease patients who displayed psychotic behaviour were recruited. Clozapine was commenced at a dose of $6.25 \mathrm{mg}$ or $12.5 \mathrm{mg}$ in the evening and this was titrated to a maximum dose of $75 \mathrm{mg}$ a day depending on response. Two patients were withdrawn because of adverse affects. Ten patients experienced a complete disappearance of psychotic symptoms and clozapine was stopped. A further fifteen patients continued treatment (mean daily dose, $42 \mathrm{mg}$ (range $12.5-75 \mathrm{mg}$ ) for a mean of 13.4 months (range 10-24 months) ). These patients remained free of psychiatric symptoms and did not display any motor deterioration. Most of the patients in this study did not require changes in their antiParkinsonian medication, although eleven were able to tolerate small increases in dose without deterioration in their mental state.

Set against these successful studies are the results of the only double blind study to date
(Wolters et al, 1990). In this small study of six patients, clozapine was titrated, over a ten day period, to a maximum dose of $250 \mathrm{mg}$ a day. This dose remained stable for ten days while the subjects' levo-dopa and or bromocriptine were increased. Clozapine was then replaced over a four day period with placebo which was continued for a further sixteen days. The evaluators were blinded but unblinded cliniclans made dosage adjustments. Only three patients completed the study as the other three patients withdrew because of adverse effects (one because of excess sedation associated with dehydration, two because of delirium). Two of the three patients that completed the study also developed mild delirium and all three experienced exacerbation of their Parkinsonism. Rather surprisingly, the authors stated that clozapine appeared to enable doses of the anti-Parkinsonian drugs to be increased without a corresponding worsening of their psychosis. It should also be noted that the high incidence of adverse effects experienced were probably caused by the relatively high doses of clozapine being used. Such adverse effects are also likely to make worthless any attempts at blinding.

Thus clozapine, in low doses, appears to be an effective medication in the treatment of psychosis in Parkinson's disease. Unfortunately, clozapine is only licensed for the treatment of schizophrenia. Moreover, clozapine prescribing is so tightly controlled by the manufacturers, Sandoz, that supplies are unlikely to be made available for non-schizophrenic illness. Therefore, first line treatment should be the careful manipulation of the patient's anti-Parkinsonian medication. If this is not successful, a trial of low dose thioridazine, sulpiride or risperidone should be considered. Occasionally, short-lived psychotic symptoms may be effectively treated with simple sedattves such as temazepam or chlormethiazole. In refractory cases, Sandoz may be contacted with a view to negotiating the use of clozapine under strict monitoring conditions.

\section{Acknowiedgements}

The authors thank Dr Ray Chaudhuri and Dr Karen Atkin for their advice on the preparation of this article.

\section{References}

Eus, C. M., Lemiens, G., PARKer, J., et al (1995) Use of apomorphine in Parkinsonian patients with neuropsychiatric complications to oral treatment. Neurology. 4.S(Suppl. 4) A251.

FActor, S. A., Brown, D., Molho, E. S., et al (1994) Clozapine: a 2-year open trial in Parkinson's disease patients with psychosis. Neurology. 44, 544-546.

FORD, B., LYNCH, T. \& GREENE, P. (1994) Risperidone in Parkinson's disease. Lancet, S44, 681. 
Meco. G.. Alessandria, A., Bonifati, V., et al (1994) Risperidone for hallucinations in levo-dopa-treated Parkdnson's disease patients. Lancet, S43, 1370-1371.

PFEIFFER, C. \& WAGNER, M. L. (1994) Clocapine therapy for Parkinson's disease and other movement disorders. American Journal of Hospttal Pharmacy. 81, 3047-3053.

QUINN. N. (1995) Drug treatment of Parkinson's disease. Brittsh Medical Journal, 310, 575-579.

RABEY, J. M., Treves, T. A., Neureud, M. Y., et al (1995) Lowdose clozapine in the treatment of levo-dopa-induced mental disturbances in Parkinson's disease. Neurology. 45. 432-434.
RAy Chaudhur, K., AbBott, R. J. \& MmLac, P. A. C. (1990) Subcutaneous apomorphine for Parkinsonian patients with psychlatric side-effects on oral treatment. Journal of Neurological and Newrosurgical Psychiatry, 84, 372-373. WolTERs, E. C., HURWITz. T. A., MAK, E., et al (1990) Clozapine in the treatment of parkinsonian patients with dopaminomimetic psychosis. Neurology, 40, 8s2-8s4.

Denise Duncan, Drug Information Pharmacist, and David Taylor, Chief Pharmacist, The Maudsley Hospital, Denmark Hill, London SE5 8AZ

\section{Psychopathic and Antisocial Personality Disorders}

\section{By Bridget Dolan \& Jeremy Coid}

The effectiveness of treatment of people with psychopathic or antisocial personality disorders is a cause for concern among many health professionals. These patients manifest severe psychological disturbance, and their behaviour can be disruptive or even dangerous. This timely book provides an overview of contemporary diagnostic concepts of psychopathic disorder, a review of natural history studies, and a comprehensive assessment of outcome studies of various treatments considered in a range of settings (the community, non-secure hospitals, secure units, Special Hospitals, and prisons).

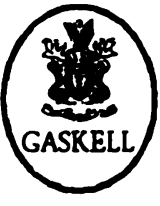

\section{$£ 20.00 \bullet 323 p p . \odot 1993 \bullet$ ISBN 0902241664}

Available from the Publications Department, Royal College of Psychiatrists, 17 Belgrave Square, London SW1X 8PG (Tel. 0171-235 2351) 